\title{
Analisi basata sugli sforzi locali della resistenza a fatica di giunzioni incollate di materiali compositi
}

\author{
S. Beretta, A. Bernasconi \\ Politecnico di Milano,Dipartimento di Meccanica, via La Masa 34 -20156 Milano, stefano.beretta@polimi.it
}

\author{
A. Pirondi, F. Moroni \\ Università degli Studi di Parma, Dipartimento di Ingegneria Industriale, viale G.P. Usberti, 181 / A - 43100 Parma
}

RIASSUNTO. Il lavoro prende spunto dai risultati di un'analisi sperimentale del comportamento a fatica di giunzioni incollate di materiali compositi laminati di elevato spessore formati da strati di unidirezionale e di tessuto di fibra di carbonio. I giunti sono stati realizzati in modo tale da saggiare l'influenza della lunghezza di sovrapposizione (da $25,4 \mathrm{~mm}$ a $110,8 \mathrm{~mm}$ ), della forma del giunto (con e senza rastremazione), e della composizione degli aderendi (sostituzione di uno degli aderendi in composito con uno in acciaio). Mediante analisi 2D elastiche con il metodo degli elementi finiti sono state ricavate le distribuzioni degli sforzi all'interno dello strato di adesivo, al fine di individuare un parametro utile alla descrizione del comportamento a fatica in termini di sforzi locali - numero di cicli a rottura. Il ruolo della fase di propagazione viene discusso alla luce di osservazioni dell'avanzamento della frattura, condotta su alcuni dei giunti testati.

\begin{abstract}
Results of fatigue tests on adhesive lap joints of thick $(10 \mathrm{~mm})$ composite laminates are presented and discussed. Specimens of different overlap length (from 25 to $110 \mathrm{~mm}$ ), different shape (with and without taper) and different materials (composite on composite, composite on steel) were fatigue tested. In order to investigate on the relationship between peak elastic stresses in the adhesive layer and fatigue life, a $2 \mathrm{D}$ structural analysis of the joints by the finite element method was performed. This analysis suggested that peak elastic stresses in the adhesive layer could be adopted as a design criterion, at least as an engineering tool for industrial applications. The role of crack propagation is also discussed, on the basis of some observations during fatigue tests.
\end{abstract}

Parole Chiave. Fatica; Giunti incollati; Sforzi locali; Materiali compositi

\section{INTRODUZIONE}

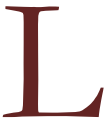

a resistenza a fatica delle giunzioni incollate di materiali compositi riveste particolare importanza per molte applicazioni in cui si renda necessario adottare la soluzione dell'incollaggio per abbinare l'efficienza meccanica dei materiali compositi con le proprietà di altri materiali, per esempio per inserti resistenti all'usura, oppure quanto dettato da esigenze costruttive o economiche (può risultare utile collegare parti in composito tra loro per ridurre i costi più stampi di forme meno complesse invece di un unico stampo più complesso - o per garantire la modularità per componenti appartenenti a piattaforme di prodotto differenti). Inoltre il fenomeno della fatica di queste giunzioni presenta aspetti peculiari legati alle caratteristiche degli aderendi in materiale composito [1].

In questo lavoro sono state analizzate alcune giunzioni incollate di materiali compositi e di materiale composito su acciaio, al fine di verificare se fosse possibile adottare un criterio di resistenza a fatica basato sui valori degli sforzi assunti localmente nello strato di adesivo, come suggerito in [2]. Una soluzione di questo tipo si è infatti dimostrata valida per interpretare la resistenza statica [3]. Sebbene in questo modo il risultato sia semplicemente una condizione limite per le 
forze trasmesse dai giunti, rinunciando a priori ad una descrizione dettagliata del fenomeno fisico (nucleazione e propagazione [4], variazioni di percorso delle fratture durante la propagazione, effetto della singolarità degli sforzi in corrispondenza delle discontinuità geometriche, come mostrato ad esempio in [5]), tale soluzione presenta l'indubbio vantaggio di prestarsi facilmente al trasferimento ad applicazioni industriali.

\section{ATTIVITÀ SPERIMENTALE}

$\mathrm{L}$

a forma e le dimensioni dei provini utilizzati per le prove di fatica sono riportati in Fig. 1. In Fig. 1 sono anche riportate le sigle utilizzate nel seguito per indicare i lotti di provini. Si tratta di quattro lotti di giunti a sovrapposizione semplice. Per tre di questi lotti gli aderendi sono entrambi di materiale composito, mentre per il restante lotto uno dei due aderendi è realizzato in acciaio strutturale ad alta resistenza S690. Il materiale composito è un laminato di $9.9 \mathrm{~mm}$ di spessore, composto da alternanze di lamine unidirezionali e di tessuto di fibre di carbonio a basso modulo immerse in matrice polimerica, la cui legge di laminazione è riporta in Tab. 1. L'adesivo utilizzato è un epossidico bi-componente ad alta resistenza (3M 9323). Lo spessore di $0.2 \mathrm{~mm}$ dello strato di adesivo è stato assicurato mediante inserimento di fili di rame di diametro calibrato disposti longitudinalmente rispetto all'asse dei provini, con eccezione dei provini LS $25,4 \mathrm{~mm}$.

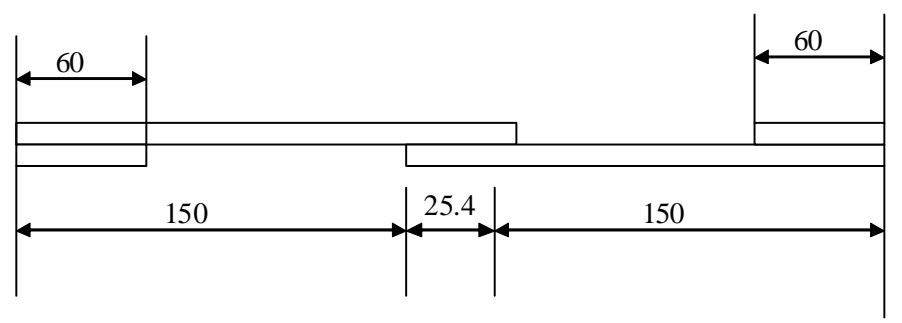

LS $24.5 \mathrm{~mm}$

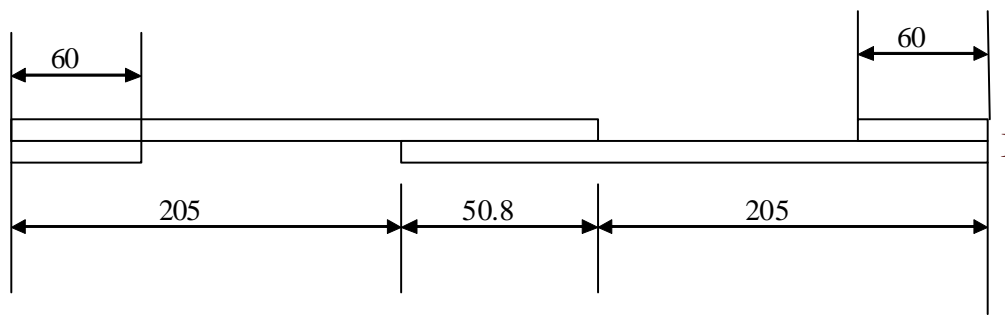

LS $50.8 \mathrm{~mm}$

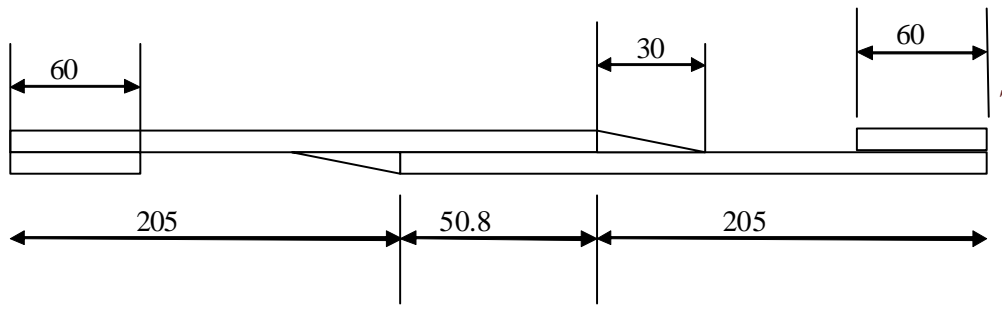

TC $110.8 \mathrm{~mm}$

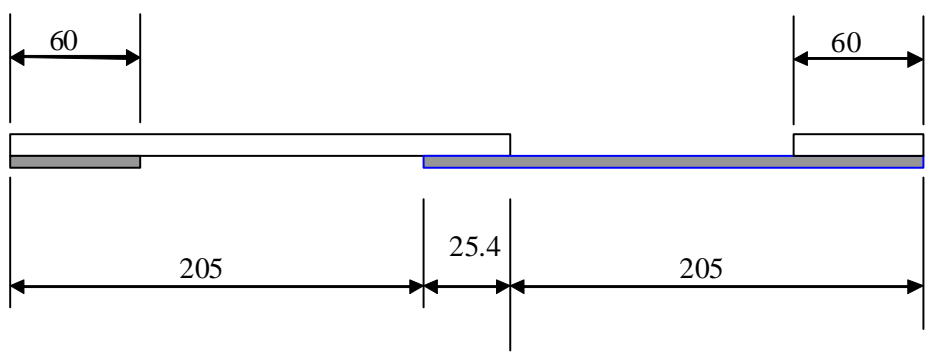

SC $24.5 \mathrm{~mm}$

Figura 1: Forma e dimensioni dei provini (non in scala). 
$\mathrm{T} 45^{\circ} / \mathrm{UD}_{5} / \mathrm{T} 0^{\circ} / \mathrm{T}_{4} 5_{2}^{\circ} / \mathrm{T}^{\circ}{ }_{2} / \mathrm{T}_{4} 5_{2}^{\circ} / \mathrm{T}^{\circ} / \mathrm{UD}_{5} / \mathrm{T} 45^{\circ}$

Tabella 1: Legge di laminazione del laminato ( $\mathrm{T}$, tessuto da $0.66 \mathrm{~mm}$ di spessore; UD, unidirezionale di $0.33 \mathrm{~mm}$ di spessore).

Per poter eseguire le prove senza introdurre componenti di flessione durante l'afferraggio dei giunti nella macchina di prova, sono stati aggiunti mediante incollaggio talloni di alluminio di spessore uguale a quello del laminato. Per i provini della serie rastremata, è stato richiesto di terminare la rastremazione con un dente iniziale di altezza $1 \mathrm{~mm}$. La geometria del dente è riportata in Fig. 2. Le prove sono state eseguite presso i Laboratori del Dipartimento di Meccanica del Politecnico di Milano, utilizzando una macchina di prova servo-idraulica Schenck Hydropuls da $250 \mathrm{kN}$ di portata. Le prove sono state condotte in un ambiente a temperatura variabile tra $23^{\circ} \mathrm{C}$ e $26{ }^{\circ} \mathrm{C}$, con il solo controllo della temperatura mediante impianto di condizionamento. Le prove di fatica sono state condotte in controllo di carico ad una frequenza di 2 Hz. Le prove sono state interrotte alla completa separazione della giunzione. Il rapporto di carico $R=F_{\min } / F_{\max }$ è stato imposto uguale a 0 .

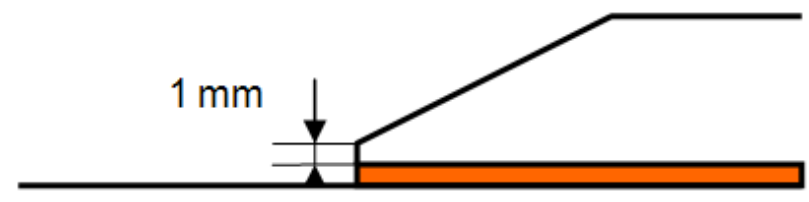

Figura 2: Dettaglio del dente al termine della rastremazione.

La caratterizzazione dell'adesivo è stata invece eseguita presso i laboratori del Dipartimento di Ingegneria Industriale dell'Università degli Studi di Parma. Le proprietà dell'adesivo sono state determinate mediante prove di trazione su un provino ottenuto colando l'adesivo in uno stampo avente forma di un provino standard ASTM 628 tipo IV e attendendone la solidificazione in forno secondo il ciclo di cura previsto dal produttore. Il provino è stato strumentato con un clip-gage e due strain-gages rispettivamente a $0^{\circ}$ e $90^{\circ}$ rispetto alla direzione del carico, in modo da poter valutare il modulo elastico E ed il coefficiente di Poisson $v$. I valori così ottenuti sono $\mathrm{E}=2300 \mathrm{MPa}, \boldsymbol{v}=0,33$.

\section{RISULTATI}

$\mathrm{R}$

iportando in un unico grafico i valori di forza massima applicata (normalizzate rispetto alla forza media necessaria per portare a rottura i giunti LS $25,4 \mathrm{~mm}$ in 100.000 cicli) e il corrispondente numero di cicli a rottura, i punti sperimentali relativi ai quattro lotti di giunti si dispongono come in Fig. 3, dove sono riportate anche le quattro curve interpolanti i risultati sperimentali secondo una legge lineare in scala doppio logaritmica. E' evidente che a seconda del tipo e della forma del giunto, nonché della lunghezza di sovrapposizione, si ottengono quattro curve distinte.

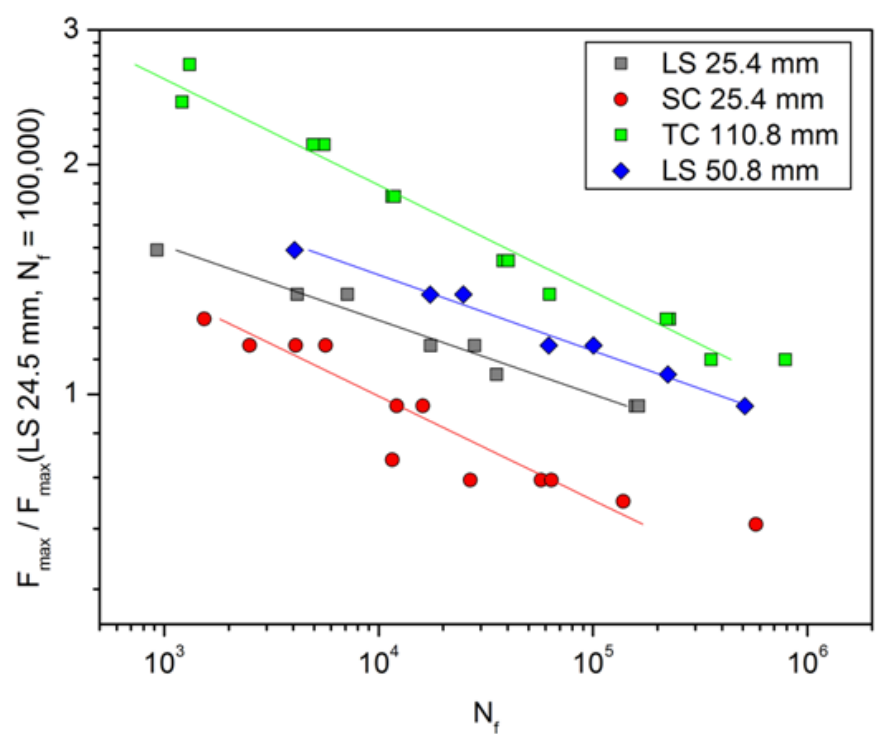

Figura 3: Curve Forze-Numero di cicli a rottura dei quattro lotti di giunti. 


\section{ANALISI DELLE FRATTURE}

A

separazione finale dei due aderendi avvenuta, l'ispezione delle superfici di frattura rivela nella maggior parte dei campioni LS 25,4 mm una zona di probabile cedimento contenuta all'interno dello strato di adesivo, che interessa tutta l'area di sovrapposizione, come mostrato in Fig. 4. Ciò invece non è avvenuto nei giunti SC 25,4 mm, in cui il cedimento è presumibilmente avvenuto sempre all'interfaccia adesivo-acciaio o adesivo-composito, come si può dedurre dall'aspetto delle superfici di frattura, tutte simili a quella riportata in Fig. 5. Nel caso dei giunti con maggior lunghezza di sovrapposizione (da $50,8 \mathrm{~mm}$ a $110 \mathrm{~mm}$ ) si è osservato una maggioranza di cedimenti riconducibili ad una fase di nucleazione in corrispondenza di uno o di entrambi gli estremi della sovrapposizione, seguita da apparente propagazione nello strato di adesivo e ulteriore propagazione interlaminare tra primo strato di tessuto e unidirezionale che ha interessato buona parte della sovrapposizione, come si può osservare in Fig. 6 per i giunti LS 50,8 mm e in Fig. 7 per i giunti TC $110,8 \mathrm{~mm}$.

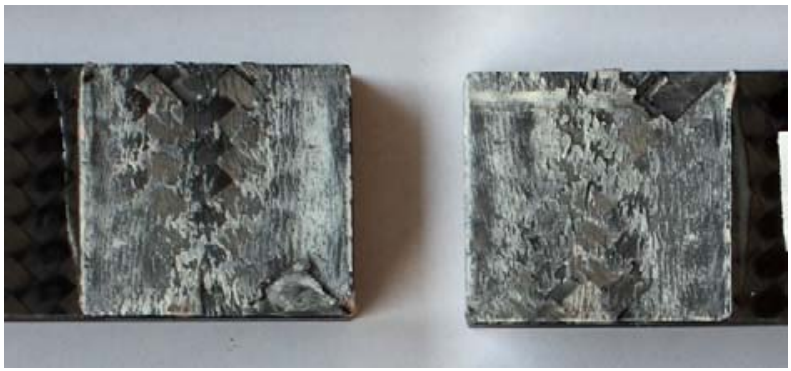

Figura 4: Superficie di frattura di un giunto LS 25,4 mm.

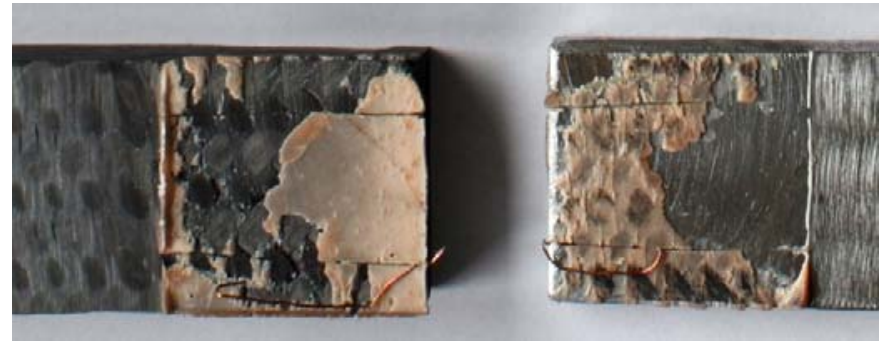

Figura 5: Superficie di frattura di un giunto SC 25,4 mm.

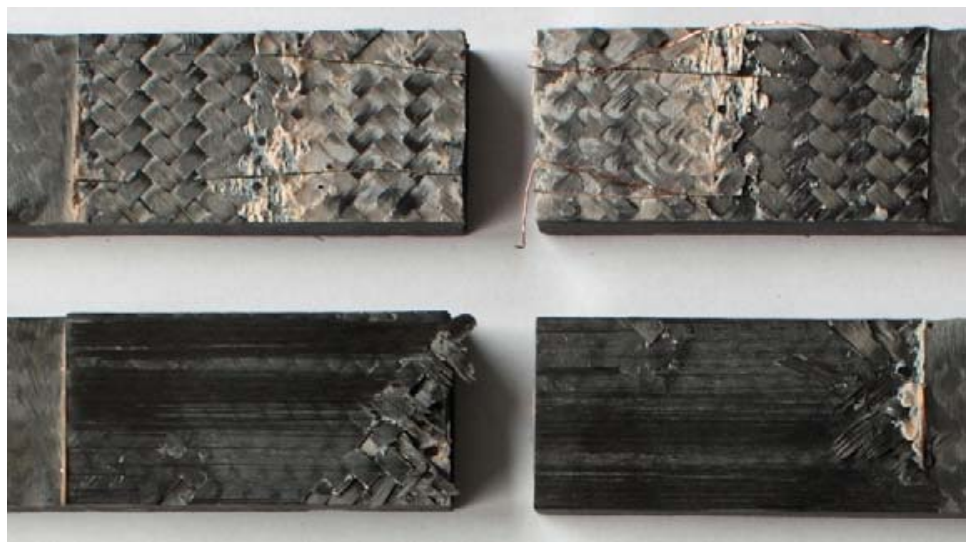

Figura 6: Superficie di frattura di giunti LS 50,8 mm .

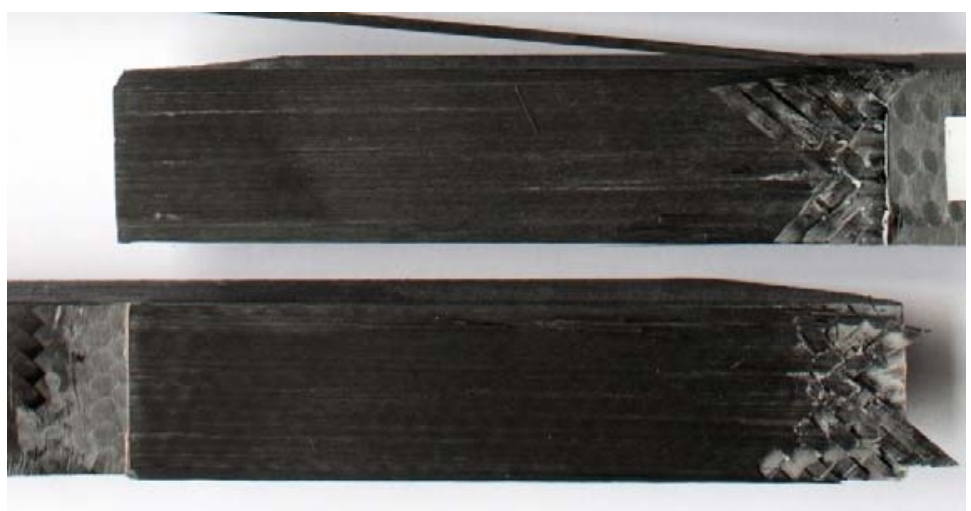

Figura 7: Superficie di frattura di un TC $110,8 \mathrm{~mm}$. 


\section{INTERPRETAZIONE DEI RISULTATI SULLA BASE DEGLI SFORZI LOCALI}

$\mathrm{I}$ giunti sono stati analizzati con il metodo degli elementi finiti, con lo scopo di determinare la distribuzione degli sforzi all'interno dello strato di adesivo. Per fare ciò, sono stati adottati modelli $2 \mathrm{D}$ in stato di deformazione piana, utilizzando per la modellazione dello strato di adesivo elementi quadratici a 8 nodi di altezza pari a $0,05 \mathrm{~mm}$, così da distribuire quattro elementi nello spessore dello strato di adesivo. Gli aderendi sono stati modellati rispettando la legge di laminazione, assegnando cioè le costanti elastiche delle rispettive lamine alle partizioni del modello operate per rappresentare fedelmente la sovrapposizione dei diversi strati.

Sono state condotte analisi elastiche lineari, adottando per lo strato di adesivo una legge elastica lineare, con i valori di modulo elastico e coefficiente di Poisson ricavati dalle prove di caratterizzazione sull'adesivo colato in massa. Le costanti elastiche delle lamine sono state assegnate sulla base dei risultati di prove di caratterizzazione meccanica eseguite presso i Laboratori del Dipartimento di Meccanica del Politecnico di Milano, utilizzando una macchina di prova elettromeccanica MTS RF 150. I valori degli sforzi tangenziali paralleli al piano dell'adesivo, degli sforzi perpendicolari allo stesso piano, dello sforzo tangenziale massimo, misurato attraverso la definizione di sforzo equivalente secondo Tresca e dello sforzo principale massimo, sono stati letti sul piano medio dell'adesivo in corrispondenza del nodo maggiormente sollecitato.

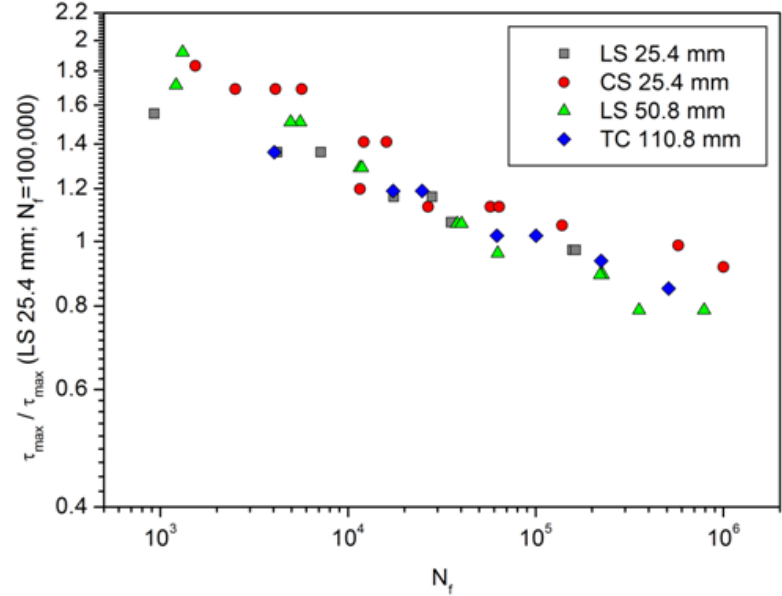

(a)

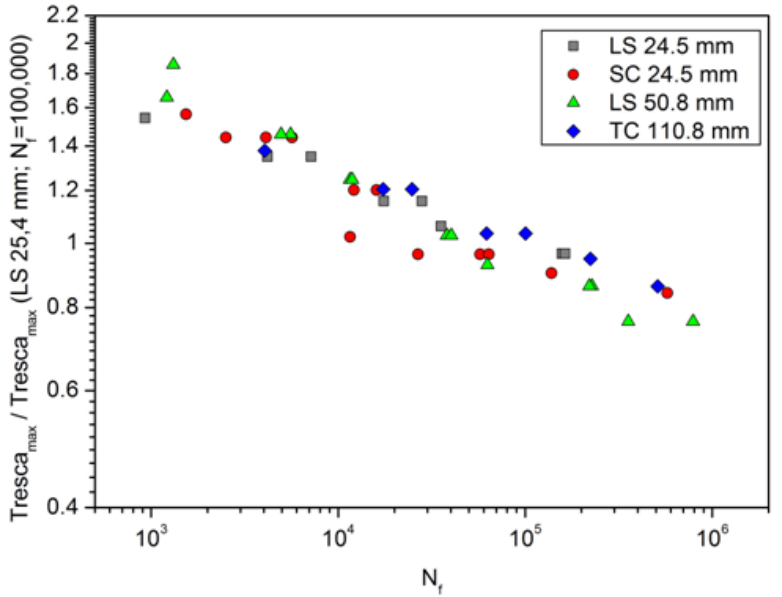

(b)

Figura 8: Diagramma numero di cicli a rottura - sforzo massimo di taglio parallelo allo strato di adesivo (a) e numero di cicli a rottura - sforzo massimo di Tresca (b), calcolati con modelli 2D agli elementi finiti.

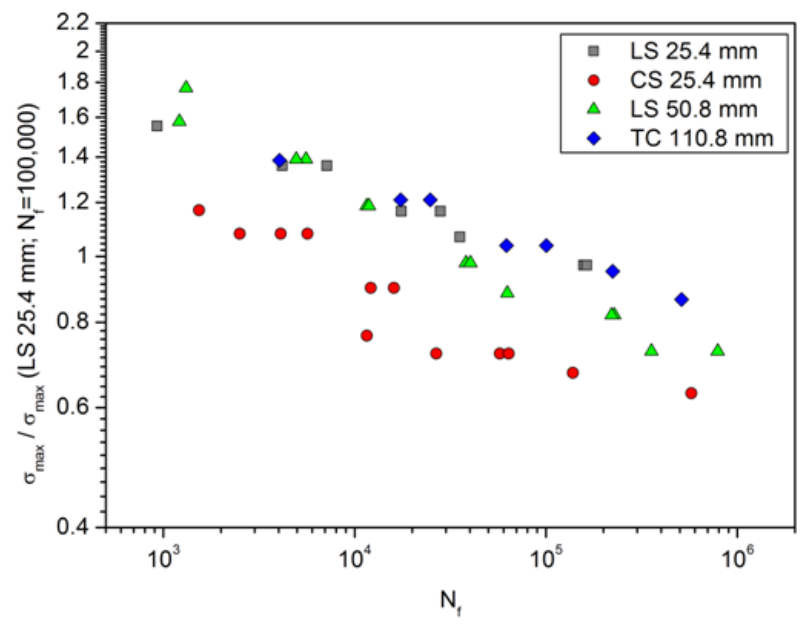

(a)

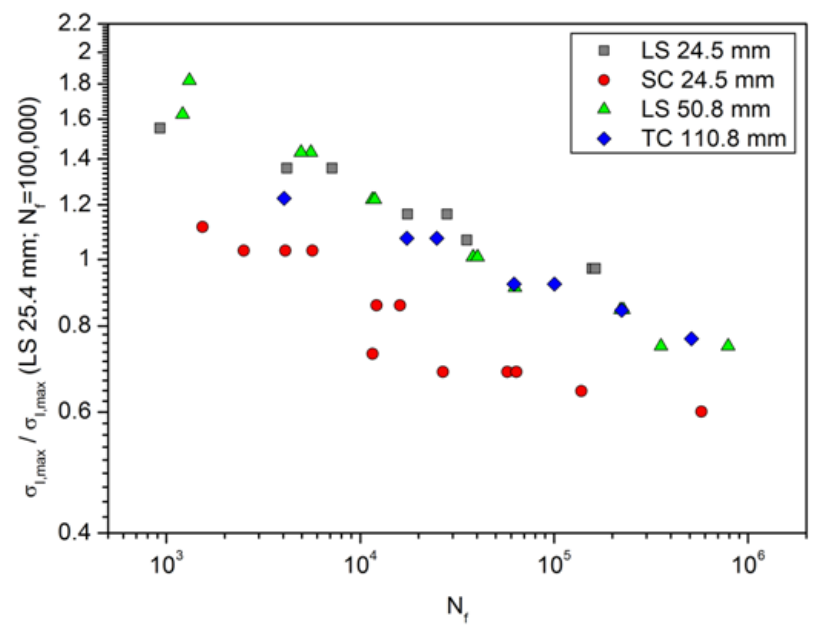

(b)

Figura 9: Diagrammi numero di cicli a rottura - sforzo massimo normale perpendicolare allo strato di adesivo (a) e numero di cicli a rottura- sforzo principale massimo (b), calcolati con modelli 2D agli elementi finiti. 
I punti sperimentali sono stati quindi riportati nei grafici delle Fig. 8 e 9, dove i numeri di cicli a rottura sono stati riportati in funzione rispettivamente dello sforzo massimo di taglio, dello sforzo massimo normale, dello sforzo massimo di Tresca e dello sforzo principale massimo. Tutte le grandezze sono state normalizzate dividendo per il valore relativo ai provini di tipo LS 25.4 mm, per una durata media di 100.000 cicli. Come si può osservare in Fig. 8a, la correlazione tra numero di cicli e sforzo massimo di taglio è migliore di quella esistente tra cicli e sforzo massimo normale (Fig. 9a), sebbene in quest'ultimo caso la maggior parte della dispersione sia da attribuire al giunto acciaio-composito, in cui si è osservato come la fattura sia propagata essenzialmente all'interfaccia tra acciaio e adesivo (Fig. 5). Il ruolo degli sforzi normali, già proposto come parametro di verifica in [7], sembra tuttavia essere meglio interpretato se si valuta la loro combinazione con gli sforzi tangenziali, attraverso la misura di Tresca. In questo caso, come è possibile osservare nel grafico di Fig. 9b, si ottiene un'ulteriore riduzione della dispersione rispetto all'adozione della sola componente di taglio. Ciò sembrerebbe suggerire l'adozione di questo parametro come criterio di verifica valido per tutti $i$ tipi di giunzione (invece, analogamente a quanto osservato per lo sforzo normale, anche lo sforzo principale massimo - Fig. $9 \mathrm{~b}-$ presenta una correlazione più bassa con il numero di cicli). Tuttavia è necessario sottolineare come questo modello, sebbene consenta un'ottima correlazione con i dati sperimentali, non tenga in nessun conto l'effettivo meccanismo di evoluzione del danno di fatica, che è caratterizzato da un ruolo predominante delle fase di propagazione, come mostrato di seguito.

\section{ANALISI DELLE FASI DI NUCLEAZIONE E DI PROPAGAZIONE}

$\mathrm{P}$ er meglio comprendere il ruolo della fase di nucleazione e le modalità di propagazione, alcuni provini (in più rispetto a quelli di cui sono riportati i risultati in Fig. 3) sono stati monitorati durante la prova di fatica mediante osservazione al microscopio ottico dello strato di adesivo affiorante sul fianco del provino. Le osservazioni sono state fatte ad intervalli regolari, sospendendo la prova e ponendo il giunto in tensione ad un carico uguale al carico massimo nel ciclo per il tempo strettamente necessario per l'acquisizione dell'immagine.

In Fig. 10 sono riportate le osservazioni dell'evoluzione del danneggiamento di un giunto LS 25,4 $\mathrm{mm}$. La prova è durata complessivamente 17200 cicli e si è interrotta per la completa separazione dei lembi del giunto. Come si può osservare dalla figura, la frattura è nucleata già a soli 500 cicli, rappresentata da una delaminazione all'interno del primo strato di tessuto sottostante l'adesivo, osservabile in corrispondenza dell'inizio della sovrapposizione degli aderendi. Questa frattura è poi propagata nella lamina e tra 12500 e 16000 cicli la frattura ha deviato al'interno dello strato di adesivo fino a quando è intervenuto il cedimento, avvenuto in corrispondenza di un avanzamento della frattura di circa $7 \mathrm{~mm}$.

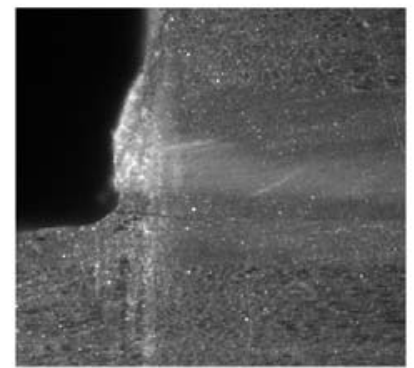

500

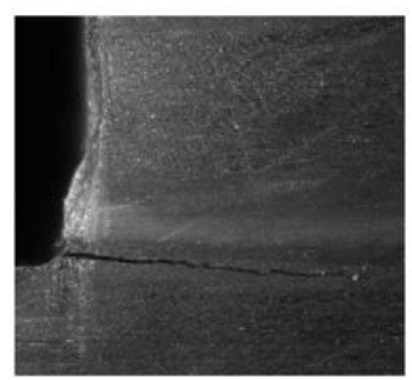

12500

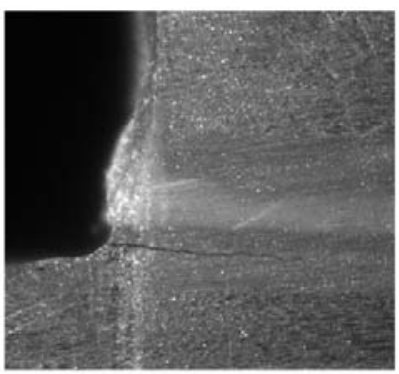

2000

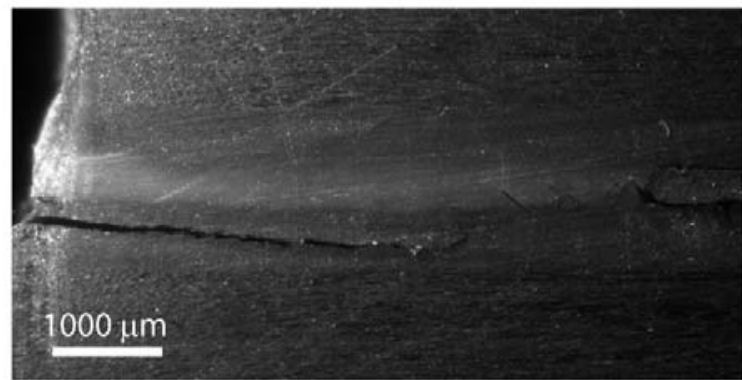

16000

Figura 10: Osservazioni dell'evoluzione del danneggiamento di un giunto da LS 25,4 mm (durata della prova: 17200 cicli). 


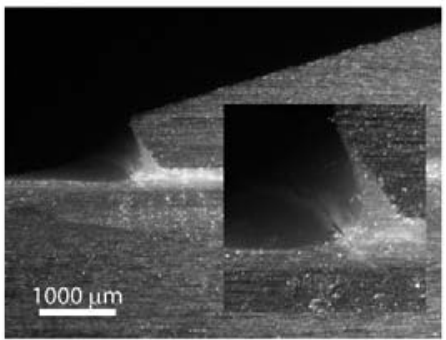

3000

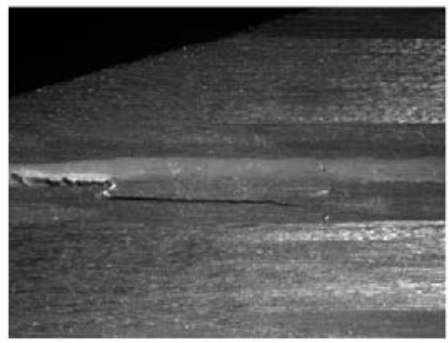

12000

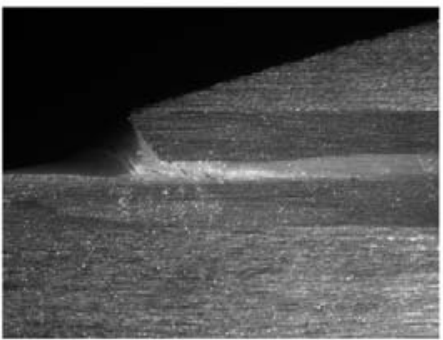

6000

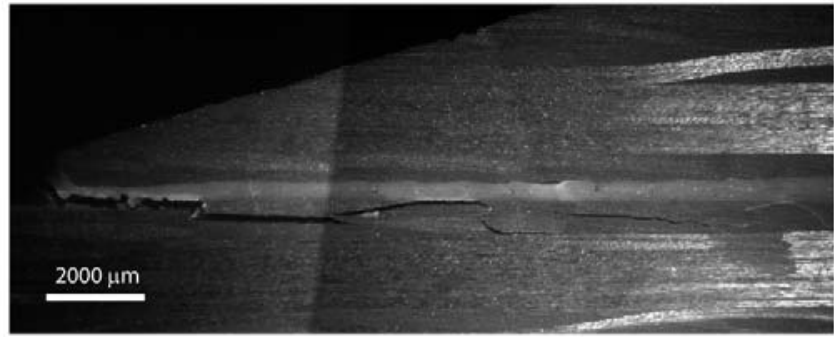

15000

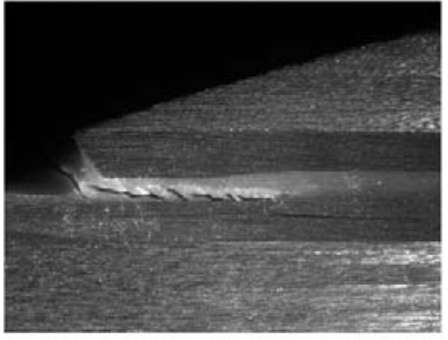

9000

Figura 11: Osservazioni dell'evoluzione del danneggiamento di un giunto da TC $110.8 \mathrm{~mm}$ (durata della prova: 18700 cicli).

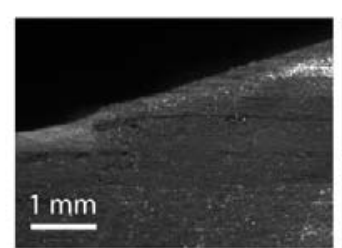

25000

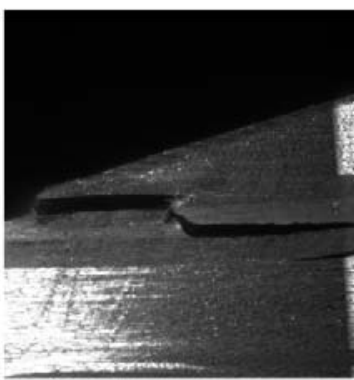

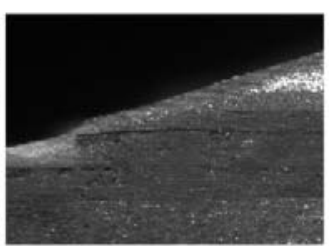

50000

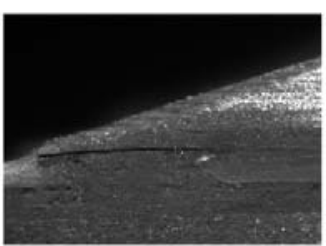

75000

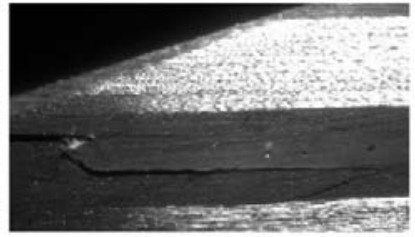

100000

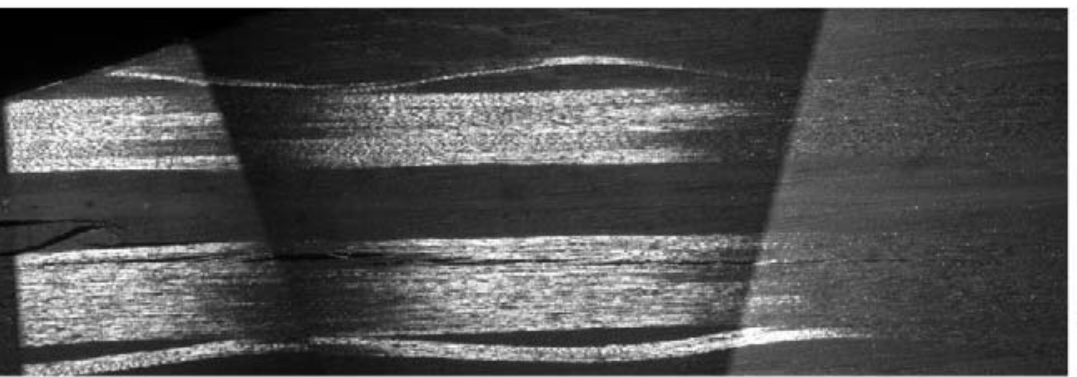

110000

Figura 12: Osservazioni dell'evoluzione del danneggiamento di un giunto da TC $110.8 \mathrm{~mm}$ (durata della prova: 140500 cicli).

Nel caso invece di un giunto TC $110.8 \mathrm{~mm}$, come è possibile osservare in Fig. 11, la fase di nucleazione è iniziata a circa 3000 cicli (su una durata complessiva della prova di 18700 cicli), con lo sviluppo di una frattura all'interno dello strato di adesivo, visibile in corrispondenza del termine della sovrapposizione rastremata. Fino a 9000 cicli la propagazione è avvenuta all'interno dello strato di adesivo, per poi interessare la lamina sottostante, con un successivo percorso di avanzamento della frattura caratterizzato da numerosi cambi di direzione, presumibilmente attribuibili alla struttura non omogenea della lamina di tessuto. Il cedimento finale è avvenuto dopo un avanzamento della frattura di più di $50 \mathrm{~mm}$ dall'estremo della sovrapposizione.

Nel caso infine di una prova condotta ancora su un giunto da $110 \mathrm{~mm}$ di sovrapposizione rastremato, ma ad un livello di forza massima inferiore, tale da determinare una durata di 140500 cicli, si è osservata una nucleazione per de laminazione in corrispondenza dell'inizio della sovrapposizione, nell'aderendo superiore (Fig. 12). A 75000 cicli il percorso della frattura ha deviato nell'interfaccia tra l'aderendo inferiore e l'adesivo, accompagnato da una contemporanea delaminazione nell'aderendo inferiore, come si può vedere dall'immagine ripresa a 100000 cicli. La propagazione ha successivamente 
interessato la lamina di unidirezionale sottostante il primo strato di tessuto, raggiungendo un'estensione di più di $80 \mathrm{~mm}$ (di cui $40 \mathrm{~mm}$ percorsi negli ultimi 20000 cicli).

\begin{tabular}{llll}
\hline Tipo di giunto & $\mathrm{N}_{\text {TOT }}$ & $\mathrm{Ni}$ & $\mathrm{N}_{\mathrm{i}} / \mathrm{N}_{\text {TOT }}$ \\
LS $25.4 \mathrm{~mm}$ & 17200 & 500 & 0.03 \\
TC $110.8 \mathrm{~mm}$ & 18700 & 3000 & 0.16 \\
TC $110.8 \mathrm{~mm}$ & 140500 & 25000 & 0.18 \\
\hline
\end{tabular}

Tabella 2: Valori della frazione di vita $\mathrm{N}_{\mathrm{i}} / \mathrm{N}_{\text {TOT }}$ spesa nella fase di nucleazione.

Mentre nel caso di giunti ad elevata lunghezza di sovrapposizione la fase di propagazione risulta sempre evidente anche ad occhio nudo in virtù degli elevati valori di apertura dei lembi della frattura, a sua volta effetto delle elevate lunghezze di propagazione, per individuare questo fenomeno nei giunti da $25,4 \mathrm{~mm}$ di sovrapposizione è stata necessaria l'osservazione al microscopio. Sebbene non siano state monitorate tutte le prove, sulla base di queste osservazioni è ragionevole concludere che l'effettivo meccanismo di evoluzione del danno di fatica sia caratterizzato da un ruolo predominante delle fase di propagazione, come riassunto in Tabella 2, in cui viene riportato per ciascun provino la frazione $\mathrm{N}_{\mathrm{i}} / \mathrm{N}_{\text {TOT }}$, dove rispetto al numero di cicli totale $\mathrm{N}_{\text {TOT }}, \mathrm{N}_{\mathrm{i}}$ corrisponde al momento in cui è stata rilevata la nucleazione della frattura. I valori riportati confermano il ruolo predominante della fase di propagazione e risultano mediamente più bassi rispetto ai valori riportati in [8], ricavati da una base molto più ampia di osservazioni, ma che si riferiscano a laminati più sottili (spessore 1,6 mm contro i 9,9 mm dei giunti oggetto di questo lavoro).

\section{CONCLUSIONI}

工

'analisi numerica mediante modelli agli elementi finiti di giunzioni incollate di aderendi realizzati in materiale composito di elevato spessore ha permesso di definire lo sforzo equivalente di Tresca quale parametro utile alla previsione del comportamento a fatica in termini di numero di cicli totale. Il valore dello sforzo equivalente è stato ricavato da modelli $2 \mathrm{D}$ e fa riferimento alla sollecitazione massima registrata in corrispondenza del piano medio dell'adesivo. L'adozione di questo parametro ha permesso di ottenere una buona correlazione con il numero di cicli a rottura, indipendentemente dalla forma del giunto, dalla lunghezza di sovrapposizione e del tipo di aderendi (limitatamente all'alternativa tra acciaio e un particolare laminato, mentre non sono state prese in considerazione leggi di laminazione differenti da quella testata).

Questi risultati sembrano suggerire che uno sforzo equivalente locale possa essere proposto come strumento di uso ingegneristico per la verifica di giunzioni incollate di materiali compositi. Tuttavia, alla luce di alcune osservazioni del comportamento locale delle giunzioni durante le prove di fatica, è stato evidenziato un ruolo predominante della fase di propagazione, che avviene sia nello strato di adesivo sia per cedimento inter- e intra-laminare. L'attività di ricerca futura si orienterà pertanto nella direzione di caratterizzare il comportamento a frattura del sistema composito/adesivo e di sviluppare modelli adeguati alla previsione della fase di propagazione in giunti incollati di materiale composito di elevato spessore.

\section{BIBLIOGRAFIA}

[1] W.C. de Goeij, M.J.L. van Tooren, A. Beukers, Materials and Design, 20 (1999) 213.

[2] A.D. Crocombe, G.Richardson, Int. J. of Adhesion and Adhesives, 19 (1999) 19.

[3] L. Goglio, M. Rossetto, E. Dragoni, Int. J. of Adhesion \& Adhesives, 28 (2008) 427.

[4] M. Dessureault, J. K. Spelt, Int. J. of Adhesion and Adhesives, 17 (1997) 183.

[5] M. Quaresimin, M. Ricotta, Int. J. of Fatigue, 28 (2006) 1166.

[6] H. Hadavinia, A. J. Kinloch, M.S.G. Little, A.C. Taylor, Int. J. of Adhesion and Adhesives, 23 (2003) 449.

[7] M. Imanaka, H. Nakayama, K. Morikawa, M. Nakamura, Composite Structures, 31 (1995) 235.

[8] M. Quaresimin, M. Ricotta, Composites Science and Technology, 66 (2006) 176. 